\title{
L'enseignement-apprentissage du français juridique en contexte francophone: approche contextuelle et interculturelle
}

\author{
Teaching and learning legal French in francophone context: contextual and \\ intercultural approach
}

\author{
Khatima El Krirh ${ }^{1}$ • (i) https://orcid.org/0000-0002-6845-2244 \\ Université de Cadix - Universidad de Cádiz \\ Avda. de la Universidad, s/n, 11405 Jérez de la Frontera, Cádiz.
}

RESUMEN

En este artículo damos cuenta de la incidencia del contexto jurídico y bilingüe en la enseñanza-aprendizaje del francés jurídico (FOS). Así, fenómenos como la diglosia, bilingüismo y bijuridismo repercuten en la configuración de un sistema jurídico. Para ello, se toma como ejemplo Marruecos como país del Magreb francófono cuyo sistema jurídico está caracterizado tanto por una dualidad jurídica (derecho islámico y derecho francés) como lingüística (árabe y francés). El análisis del corpus representativo revela que las asimetrías conceptuales entre dos sistemas jurídicos expresados en lengua francesa solo se pueden enseñar y aprender aplicando un enfoque contextual e intercultural.

Palabras clave: francés jurídico (FOS); Bijuridismo; Diglosia; Interculturalidad; Derecho islámico; Derecho francés.

\section{ABSTRACt}

In this article we report on the impact of the bijuridic and bilingual context on the teaching - learning of legal French (FOS). Thus, phenomena such as diglossia, bilingualism and bijuridism have an impact on the configuration of a legal system. For this purpose, we take as an example Morocco as a country of the French-speaking Maghreb whose legal system is characterized by a legal duality (Islamic law and French law) and a linguistic duality (Arabic and French). The analysis of the representative corpus reveals that the conceptual asymmetries between two legal systems expressed in the French language can only be taught and learned by applying a contextual and intercultural approach.

Keywords: legal French (FOS); Bijuridism; Diglossia; Interculturality; Muslim Law; French Law.

\section{Introduction}

Le Droit est avant tout un phénomène culturel et social puisqu'il témoigne des relations sociales, économiques et personnelles dans une société donnée. Cela veut dire que l'appréhension d'une langue juridique -francophonemarquée par le bijuridisme et le bilinguisme n'est pas toujours réalisable à cause du problème d'équivalence culturelle et linguistique. En effet, afin de mener à bien cette étude nous avons choisi le Maroc, l'un des pays du Maghreb francophone, comme un exemple de ces deux derniers phénomènes qui influencent non seulement la langue juridique arabe mais aussi le langage juridique français. 
Pour comprendre le bilinguisme et le bijuridisme au Maroc, il faudrait rappeler en amont que le français fut adopté au Maroc à la faveur du protectorat en 1912 (Traité de Fès). Dès lors, le français devint la langue officielle des institutions jusqu'au processus d'arabisation intensifié dans les années 70 auquel ont été soumis les pays du Maghreb. De suite, le droit français conflue avec le droit musulman (bijuridisme) et le français cohabite avec l'arabe (bilinguisme). Ainsi, le dualisme juridique -ou bijuridisme - et le bilinguisme ont provoqué une nécessité incontestable de traduction du langage juridique français vers l'arabe dans le but de préserver et garantir la diffusion des normes juridiques erga omnes dans les deux langues.

Dans le contexte que nous venons de décrire, la difficulté d'apprentissage du français juridique relève de la nature du dualisme juridique. Ceci contraint les étudiants et les professionnels du Droit à appréhender parallèlement la terminologie juridique du droit muslman diffusée en langue française et la terminologie juridique du droit français en langue française ${ }^{2}$.

En effet, l'objectif principal dans cet article est de montrer la problématique de l'enseignement -apprentissage du français juridique dans un contexte bilingue et bijuridique tel est le cas du Maroc. Le choix de ce contexte bilingue arabe-français est due notamment à l'absence de recherches abordant cette problématique.

Quant à la structure, cette étude s'articule en trois chapitres. Dans la première partie nous nous pencherons sur les notions de diglossie, bijuridisme et bilinguisme suivies de celles de français juridique et français de spécialité. Ensuite, nous exposerons les particularités du système juridique marocain où l'utilisation de deux langues ${ }^{3}$ pour diffuser et légiférer (l'arabe et le français) est maintenue.

Dans la troisième partie nous ciblons l'analyse du corpus parallèle (Moudawana ou code de famille marocain /code civil français). Dans cette analyse nous allons dépouiller les concepts juridiques d'origine islamique en langue française ainsi que leurs homologues en français juridique hexagonale. En ce sens, l'analyse descriptivecontrastive pour classer les termes devient une stratégie d'utilité pédagogique qui nous aidera à faire ressortir les asymétries conceptuelles et syntaxiques entre les deux systèmes linguistiques/juridiques.

Finalement, le corpus représentatif nous permettra d'aboutir à des conclusions qui feront émerger la problématique de l'appréhension du français juridique dans l'enchevêtrement bilinguisme/ bijuridisme. Concrètement, la maîtrise des traits distinctifs entre les concepts juridiques du Droit islamique et ceux du Droit français transposés en langue française est décisive pour affronter les exigences du marché de l'emploi dans le milieu juridique arabofrancophone.

Dans le cadre de notre expérience dans l'enseignement du français sur objectifs spécifiques, particulièrment le français juridique, l'approche contextuelle et culturelle favorise son acquisition du fait qu'elle est orientée aux besoins des apprenants qui visent un usage professionnel effectif de la langue juridique cible.

2 Les études universitaires de Droit au Maroc sont dispensées en deux licences bien distinctes: Droit français et Droit arabe

3 Une de fait (le français) et l'autre de droit (l'arabe) 


\section{Diglossie, bilinguisme et bijuridisme}

Maints auteurs ont défini la diglossie comme "un état particulier de langue dans lequel une communauté ou un groupement humain recourt à deux langues différentes ou à deux variétés de la même langue en fonction de situations de communication différentes" (Azouzi, 2008: 42).

Pour Frank Neveu (2004) la diglossie représente "une situation de bilinguisme étendue à l'ensemble d'une communauté linguistique au sein de laquelle s'observe l'usage de deux langues dans des circonstances précises de la vie sociale" (Neveu,2004: 57, cité par Azouzi, 2008 :42) ; ou bien en tant qu'une "configuration dans laquelle deux variétés, l'une haute, l'autre basse, de la même langue sont en usage dans une société avec des fonctions socioculturelles différentes mais complémentaires"; “ la diglossie n'est pas que deux variétés d'une même langue mais aussi de deux langues opposées en distribution fonctionnelle complémentaire" (Charles Ferguson :1959, Fishman: 1971 cités par Mabrour, 2007: 3).

De son côté, Bres (2001: 92, cité par Azouz, 2008: 43) relève qu'un contexte conflictuel est à l'origine du phénomène de la diglossie qui touche également à des questions identitaires dans la détermination de langue dominante et langue dominée engendrant des stéréotypes linguistiques et au -delà identitaires présents dans plusieurs textes. De ce sens, Azouzi (2008) souligne que le Maghreb connaît deux types de diglossie, une diglossie entre l'arabe classique en tant que langue officielle d'enseignement et l'arabe dialectal, variété basse utilisée dans la vie quotidienne (Azouzi, 2008: 43).

Le deuxième type de diglossie est celui que l'on appelle "l'alternance" entre l'arabe dialectal et la langue française générant ainsi une situation de plurilinguisme (Azouzi, 2008: 43). Telle affirmation est partagée par Bianchini (2007) qui conclut, en référence au Maghreb, que les trois pays (Maroc, Algérie et Tunisie) "sont confrontés à un plurilinguisme endémique surchargé du phénomène de diglossie, voire triglossie, et de l'alternance codique" (Bianchi, 2007 cité par Azouzi, 2008: 43). Pour ces raisons, certains linguistes défendent l'insuffisance du concept de «diglossie» en optant pour d'autres termes plus représentatifs tels que «triglossie» ou «polyglossie».

Dans l'application de ce qui précède, au Maroc l'arabe standard est remplacé à l'oral par l'arabe dialectal (le Darija). Cette pratique donne lieu à ladite "alternance entre les deux variantes de l'arabe: l'arabe dialectal marocain et l'arabe standard", une alternance qui enchaîne une situation de diglossie désignée par Fergusson comme Low Variety et High Variety (cité par Mabrour, 2007:3).

D'un autre point de vue, la notion de diglossie que nous venons de définir ne doit pas être confondue avec celle du bilinguisme. Dans ce dernier, il s'agit de l'utilisation par un locuteur de deux langues différentes et non de variétés de la même langue. En effet, le bilinguisme voire plurilinguisme du contexte marocain est marqué par l'arabe dialectal, l'amazighe et l'arabe standard ${ }^{4}$ selon la Constitution de 2011.

4 Article 5.L'Arabe demeure la langue officielle de L'Etat. L'Etat oeuvre à la protection et au développement de la langue arabe, ainsi qu'à la promotion de son utilisation. De même, l'Amazigh constitue une langue officielle de L'Etat, en tant que patrimoine commun de tous les marocains sans exception. Une loi organique définit le processus de mise en oeuvre du caractère officiel de cette langue, ainsi que les modalités de son intégration dans l'enseignement de dans les domaines prioritaires de la vie publique, et ce afin de lui permettre de remplir à terme sa fonction de langue officielle. 
Pourtant, malgré le fait que le bilinguisme français-arabe ne soit plus mentionné dans la Constitution marocaine comme langue officielle, la langue française y est toujours présente puisqu'elle demeure privilégiée comme première langue étrangère, langue seconde généralisée. En tout cas, ce qui nous intéresse ici c'est de mettre davantage l'accent sur le fait que " le français et l'arabe classique (ou moderne) se disputent le statut de langue véhiculaire et sont en concurrence dans le système éducatif, les administrations de l'État, les médias et l'économie du pays" (Trescases, 2012: 123). D'ailleurs, la langue française s'y impose en tant que langue du marché de l'emploi étant non seulement comme véhicule des secteurs économiques mais aussi comme un instrument de la promotion sociale (Himer 2000; Mabrour 2007, Soussi 2015).

Comme nous l'avons indiqué au début de cet article, ce fut à partir de l'Indépendance que le Maroc et les autres pays du Maghreb ont connu un processus d'arabisation intensifié ayant un retentissement immédiat sur la langue utilisée pour légiférer. De ce fait, l'expression de droit en français a été endiguée au profit des autres langues dites officielles (Arabe et Tamazight) conformément aux dispositions marocaines. Dans celles-ci, le traitement du français comme une langue étrangère se heurte à la praxis, notamment dans le milieu judiciaire où les conditions pour postuler en tant qu'expert judiciaire en attestent ${ }^{5}$. Ainsi, l'exercice de cette profession requiert un certificat de nationalité marocaine présupposant la maîtrise de la langue arabe voire standard/classique par ces professionnels. Paradoxalement, en cas de difficulté pour s'exprimer en arabe devant le juge, le législateur, dans l'article 65 du Code de la procédure civile marocain, reconnaît le droit de cet expert à un interprète (de langue arabe désormais langue officielle des tribunaux marocains). Sans doute, cet exemple dévoile le statut ambigu de la langue française dans les textes de lois marocains.

Cependant, ce qui est indubitable c'est que le bilinguisme juridique de fait arabe-français est à fleur de peau à tout niveau de production législative ${ }^{6}$ car l'expression du droit au Maroc est faite en langue française bien qu'elle n'ait acun statut officiel. En outre, grâce à ce bilinguisme de fait, tout apprenant du Français juridique peut se contenter parfois d'une transposition littérale puisque le français est à la base de plusieurs textes législatifs marocains.

\section{L'enseignement-apprentissage du français juridique}

\subsection{Enjeux de l'enseignement apprentissage du français juridique comme langue de spécialité}

Avant d'aborder les particularités de l'enseignement du français juridique, il est pertinent de souligner les points suivants concernant la méthode d'apprentissage d'une langue de spécialité. Dans un premier temps, la méthodologie était axée sur l'apprentissage des listes de vocabulaire (Vocabulaire Général d'Orientation Scientifique,1971) comme l'indique Lehman (1993: 91-92 cité par Toullelon,2016: 6) en distinguant trois

5 Au Maroc les formations techniques sont dispensées en langue française. Cela veut dire que la majorité de ces experts s'expriment en langue française.

6 Il suffit de consulter le site www.adala.ma pour apprécier ce bilinguisme juridique. Sur ce site tous les textes légaux sont publiés dans les deux versions: arabe et française 
niveaux : le premier se consacre aux bases de la langue usuelle alors que le deuxième s'attache au français de tronc commun scientifique où était utilisé l'ouvrage évoqué plus haut.

La dernière étape serait un niveau de perfectionnement dans les langues spécialisées tranchées par discipline/domaine de spécialité. Par conséquent, l'aspect communicatif était laissé de côté pour traiter uniquement le vocabulaire scientifique, technique, commercial etc. Or, dans le contexte professionnel réel, la compétence en langue de spécialité ne se limite pas à une compétence lexicale ou purement linguistique. Pour comprendre un texte de spécialité, dans ce cas juridique, il faudrait être plutôt un étudiant de droit qu'un apprenant d'une langue étrangère vu que les compétences linguistique, pragmatique et textuelle sont indissociables.

Ultérieurement, le paradigme de l'enseignement de langue de spécialité se serait focalisé sur l'enseignement sur objectif spécifique (de l'anglais language for special purpose) dont l'importance est octroyée au savoir-faire de spécialité. Il s'agit donc d'un processus d'enseignement-apprentissage marqué par la convergence du vocabulaire scientifique, le contexte professionnel et l'intersection de langue commune et langue de spécialité.

Dans cette optique, une analyse des besoins du public-cible est requise avant d'envisager les objectifs spécifiques de la formation. À cet égard, Binon (2000) souligne que l'enseignement basé sur le savoir-faire est désormais focalisé sur les situations-problèmes où l'on doit mobiliser ses connaissances spécialisées en plus des compétences discursives, stratégiques et linguistiques. Cela représente donc "un défi intellectuel pour l'apprenant qui doit réfléchir en langue étrangère et ne peut plus se contenter d'imiter et de reproduire" (Binon 2000: 29 cité par Letafati et Zarei, 2016: 94). La même idée est manifestée par Cuq (2003):

Le FOS est né du souci d'adapter l'enseignement du FLE à des publics adultes souhaitant acquérir ou perfectionner des compétences en français pour une activité professionnelle ou des études supérieures. (...) L'objectif de la formation linguistique n'est pas la maîtrise de la langue en soi mais l'accès à des savoir-faire langagiers dans des situations dûment identifiées de communication professionnelles ou académiques. (Cuq, 2003: 109-110, cité par Toullelon, 2016: 6)

Par rapport au processus d'acquisition de ces savoir-faire dont parle Cuq, il est important de distinguer ceux d'une langue de spécialité juridique et ceux qui appartiennent à une langue de spécialité technique. Il va de soi qu'apprendre le fonds terminologique ne saurait surmonter les difficultés notionnelles dans une langue juridique comme c'est le cas des langues techniques. La langue juridique appartient au domaine des sciences sociales, elle est créée et acquise dans une société qui évolue différemment à une autre comme l'a declaré Gémar (1980) :

Les langues techniques sont probablement les seules dont on puisse retenir aux fins d'enseignement, le fonds terminologique, généralement univoque quels que soient le lieu et la langue employée. Il n'en va pas de même pour les sciences sociales, polysémiques par définition puisque leur discours s'appuie non seulement sur des relations objectives vérifiables mais sur l'étude de l'activité humaine, variable à l'infini. (Gémar, 1980:886)

Nous sommes en accord avec l'affirmation de Gémar car c'est par le biais de cette évolution sociale que le système juridique emprunte la langue en sorte qu'un terme juridique soit exprimé sous un mot particulier dans une communauté linguistique déterminée (Terral: 2004). En effet, la problématique de la bonne maitrise de la terminologie juridique est essentiellement puisque " le rapport entre mot et concept n'est pas le même dans toutes les langues juridiques" (Sacco,1987: 850 cité par Terral, 2004: 878). Autrement dit, l'accès au savoirfaire du langage juridique exige une bonne connaissance préalable du contexte social et culturel où l'on enseigne et, de ce fait, la méthode d'enseignement devrait cerner les nuances conceptuelles auxquelles enseignant et apprenants seront confrontés. 
De même, il faut se rappeler que quand nous parlons du français juridique nous ne faisons pas référence exclusive au français juridique hexagonale mais aussi au français juridique utilisé dans les pays francophones, un concept qui a été conçu par Ben Achour (2007: 136) sous l'expression "être de culture juridique francophone ". C'està-dire que " par le français on accède au droit français, au droit communautaire et aux droits occidentaux, aux droits africains, et asiatiques, et également avoir un deuxième accès au droit musulman des écoles, aux droits coutumiers locaux ou régionaux, au droit national et aux droits des pays arabes ". Cette définition que nous jugeons pertinente est au coeur de l'approche contextuelle en enseignement du français juridique où il faudrait envisager les scénarios suivants:

a. L'enseignement du français juridique s'effectue dans un même système juridique véhiculé par plusieurs langues (Suisse et Belgique) dont le français est une des langues officielles.

b. L'enseignement du français juridique est mené dans plusieurs systèmes juridiques dans un contexte plurilingue (les pays du Maghreb, Moyen-Orient et Canada) où les lois sont diffusées aussi en langue française.

En effet, si nous comparons les deux situations nous constaterons que dans le premier contexte il serait moins complexe d'enseigner le français juridique que dans le deuxième étant qualifié ce dernier par plusieurs auteurs comme «bijuridique » (Beaupré, 1987: 206 ; Gémar, 1995: 151 cités par Terral 2004 :878) où un recours au droit comparé par l'enseignant s'avère indispensable.

\subsection{L'enseignement du français juridique : démarche contextuelle et interculturelle}

Le Droit, comme nous l'indiquions, est un fait culturel qui produit des énoncés particulièrement connotés par leur contexte socioculturel. De là, mettre l'accent sur le pilier interculturel dans l'enseignement-apprentissage du langage juridique français (francophone) est une nécessité fonctionnelle. Cependant, les approches anthropologiques, psychologiques etc., n'ont pas défini nettement ce qu'on l'entend par « culture »; pour autant nous allons introduire de brèves définitions de deux termes qui nous intéressent dans cette étude: culture et interculturalité.

Du point de vue ethnografique, De Carlo (1998: 34, cité par Roura, 2014: 52) annonce que "la culture est cet ensemble complexe qui comprend la connaissance, les croyances, l'art, la morale, le droit, les moeurs et toute capacité et habitude acquises par l'homme comme membre d'une société "; cette affirmation semble désigner tout ce qui est créé et acquis en société. Par contre, l'interculturalité représente l'idée d'action entre les participants dans un acte communicatif où on l'on envisage une résolution aux problèmes éducatifs ou sociaux issue d'une "confrontation de valeurs culturelles". C'est-à-dire, "l'interculturel désigne alors un certain type de relation entre les membres de cultures différentes" (Roura, 2014: 53). De cette façon, pour que le dialogue interculturel ait lieu, il est indispensable que "les valeurs et les formes de vie de chaque groupe soient valorisées" (Roura, 2014: 54).

Il s'ensuit qu'enseigner une langue juridique se traduit donc par mettre en valeur la culture juridique visée, connaître ses valeurs sociales et religieuses sous-jacentes. Sous ces paramètres, développer les compétences communicatives interculturelles des apprenants et des professionnels présente un intérêt capital. Ainsi, la reconnaissance et l'assimilation dans une démarche interculturelle leur permettra de faire la liaison entre les concepts complémentaires de langue-pensée-culture (Bourdieu, 2001; Lussier, 2009 cités par Amireault, 2012: 52). 
Dans le même ordre d'idées, l'approche interculturelle nous mène à l'application de la démarche contextuelle où l'on assure l'apprentissage des fonctions du langage en même temps que l'on développe son utilisation appropriée. Cet apprentissage est axé sur des connaissances antérieures sans oublier l'élément culturel dans la mesure où la compétence interculturelle articule l'ensemble des savoirs concernant l'acquisition de la culture juridique d'arivée (éléments historiques, religieux, sociaux, etc.).

C'est dans cette démarche contextuelle que les apprenants sont appelés à rechercher les significations, à comprendre le contenu et le contexte étant donné que la relation entre les dimensions linguistiques et culturelles dans la langue juridique est caractérisée d'« indissociable »(Castellotti \& Moore, 2011: 243 cités par Amireault, 2012: 53).

En revenant sur la terminologie juridique, les objectifs sont, dans l'opinion de Gémar (1980), d'amener l'étudiant à un niveau de connaissance fonctionnel de la terminologie juridique et de l'aider à acquérir en même temps une connaissance notionnelle nécessaire et suffisante. En effet, les apprenants de la langue juridique cible doivent assimiler les concepts de LS2 (langue juridique contextualisée) ${ }^{7}$ inconnus dans LS1(langue juridique de l'hexagone) ${ }^{8}$ et les intégrer dans LJA (langue juridique d'arrivée). Cela serait en fonction des conditions particulières dans lesquelles ila exerceraient leur activité (texte juridique de portée locale, régionale, nationale; document de droit privé ou public; loi, contrat, jugement, article de revue...).

Compte tenu de ce qui précède, nous nous intéressons à l'analyse comparative des concepts juridiques liés à la culture juridique d'arrivée, dans ce cas le droit marocain d'expression française que nous aborderons dans le chapitre suivant.

\section{Contexte juridique et corpus}

\subsection{Droit français et droit musulman au Maroc}

Pour Damette (2007: 8) “l'enseignement/apprentissage du français juridique présente une particularité par rapport à d'autres cours de français à visée professionnelle: la langue du droit ne véhicule pas que des techniques, mais plus fondamentalement, un système idéologique, des choix de sociétés, des valeurs".

En accord avec Damette, avant de maîtriser la terminologie juridique nous devons connaître d'abord le contexte juridique marocain en examinant les sources du droit opérantes au Maroc pour passer ensuite au dépouillement non exhaustif mais représentatif de quelques termes et/ou collocations juridiques.

Afin d'illustrer le dualisme du langage juridique français au Maroc, nous devons exposer en amont les trois sources principales du Droit marocain dans le tableau ci-dessous:

7 Ce serait le français juridique enseigné dans un contexte bijuridique-bilingue

8 Dans notre cas le français juridique enseigné en France 


\begin{tabular}{|c|c|c|}
\hline Sources classiques & Sources modernes & Source interprétatives \\
\hline La religion (Droit musulman) & Conventions internationales & $\begin{array}{l}\text { La jurisprudence } \\
\text { (الإجتهاد القضائي) }\end{array}$ \\
\hline & La Constitution 2011 & Doctrine (الفقه) \\
\hline \multicolumn{3}{|c|}{ La loi } \\
\hline Le droit coutumier & 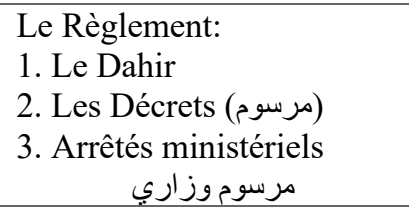 & \\
\hline
\end{tabular}

Tableau 1. Le classement de sources législatives

Premièrement, sur le plan des sources classiques ou traditionnelles se situe la religion, dans ce cas islamique, une source dont nous ne pouvons parler que dans un État confessionnel. En d'autres termes, un État qui prend en considération la religion au niveau de son droit positif (القانون الوضعي) alors que dans un État laïque (دولة علمانية) la religion n'est pas reconnue en tant que source de droit positif.

Ainsi, la religion comme source de droit appelle à un ensemble de dispositions (les règles) religieuses: la loi islamique- charria (الثريعة) basée sur le Quran (parole de Dieu) et la Sounna "(les dires ou Hadith et les faits du prophète Mohammed)" (Bellouch, 2014: 15). Lorsqu'un fait n'est pas traité dans le Coran ou la Sounna, “ un appel au consentement unanime de la communauté musulmane (Ijmâa, الإجماع)" est fait, mais si ce dernier n'est pas agréé, on recourt à l'analogie ou Al Kiyas. Cette source est appliquée, comme nous verrons dans le chapitre suivant, en matière du statut personnel (الأحوال الثخصية), droit familial (قانون الأسرة) et successoral (المير اث) (Bellouch, 2014: 16-20).

Par ailleurs, le droit coutumier est également connu par Alorf (العرف), occupant le deuxième rang en tant que source traditionnelle. Il s'agit alors d'une règle de droit non écrite résultat des pratiques sociales anciennes et spontanées des citoyens dont la répétition et la prolongation dans le temps les conditions essentielles pour devenir une source de droit. Cette source avait été reconnue sous le protectorat en tant que juridiction propre aux berbères de la montagne exercée par les tribunaux au premier degré et les Caïds en voie de recours devant les tribunaux d'appel coutumiers (Magnard, 1990: 3). Néanmoins, de nos jours, le droit coutumier n'est reconnu que pour les pratiques entre les commerçants.

Deuxièmement, par rapport aux sources modernes, nous distinguons la Loi émanant du pouvoir législatif

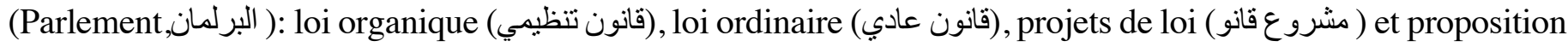
de loi (مقترح قانون) ; loi - cadre (قانون إطار) et les règlements qui sont des textes provenant du pouvoir gouvernemental ou administratifs (Roi, Chef du gouvernemet, Ministres etc.). Parmi ceux-ci nous citons le Dahir (règle juridique émanant du Roi), le Décret promulgué par Chef de gouvernement, Décret-loi (promulgué par le Roi) et les Arrêtés élaborés par une autorité subordonnée au Chef de gouvernement notamment les ministres.

Troisièmement, les sources interprétatives appelées également sources du Droit objectif. D’une part, nous citons la jurisprudence constituée par une série de décisions rendues par les différentes juridictions lorsqu'il n'y a pas

9 “On est en présence de l'ijmaa lorsque la communauté musulmane guidée par ses savants, affirme que telle est la règle de droit. Tout se passe comme si cette communauté avait été inspirée par Dieu [...]. Le Kiyas est un procédé par lequel une règle posée par un texte - verset, hadith ou ijmaa - est appliquée à des cas non compris dans ces textes, mais commandée par la même raison (illa)" (Bellouch,2014 :16) 
de normes juridiques pour trancher le litige. Ultérieurement, ces décisions sont appliquées par les juges à des cas identiques devenant ainsi une norme de droit. Or, quand un tribunal fait une nouvelle interprétation pour un cas semblable on parle d' «un revirement de jurisprudence».

D'autre part, la doctrine est l'ensemble des opinions et commentaires des juristes, professeurs et chercheurs concernant les règles juridiques, leur absence, critique et suggestions qui peuvent modifier des lois ou règles de droit dans l'avenir.

Il est important de mentionner que le vif de notre sujet est l'influence de la culture juridique islamique dans le système de droit privé et public au Maroc d'autant plus que la plus profonde influence est marquée dans les règles de droit de famille (Al-moudawana) et celles du Statut personnel.

Dans la même perspective, Belllouch (2014) manifeste que la plupart des lois marocaines "sont d'inspiration française mais l'impact du droit musulman, sur ces textes est une réalité. Il suffit de consulter par exemple le code des obligations et contrats" ${ }^{10}$ (Bellouch, 2014:10), le code foncier ${ }^{11}$, le code pénal ${ }^{12}$ et de la finance islamique $^{13}$, tous inspirés des écoles religieuses: Malékite et Hanéfite et rarement de la Chaféite. C'est pour cette raison que connaître l'organisation judiciaire liée à ce contexte s'avère prioritaire pour acquérir une terminologie juridique contextualisée.

À cette fin, aborder les deux types de juridictions au Maroc (de droit commun et les juridictions spécialisée) semble d'une importance incontestable.

Dans les juridictions de droit commun, les Tribunaux de première instance constitués en plusieurs chambres, connaissent en premier ressort de toutes les affaires non attribuées à d'autres juridictions. En deuxième degré, les Cours d'appel connaissent des recours rendus contre les décisions des tribunaux de Première instance.

10 Code des obilgations et contrats marocain:

Article 484 « est nulle entre musulmans la vente de choses déclarées impures par la loi religieuse» .

Article 870 « entre musulmans la stipulation d'intérêt est nulle et annule le contrat »

11 Code foncier marocain :

Article 10 «" [...] $2^{\circ}$ Le copropriétaire, sous réserve du droit de chefâa de ses copropriétaires, lorsque ceux-ci se trouvent dans les conditions requises pour l'exercice de ce droit ; $3^{\circ}$ Le bénéficiaire de droits réels énumérés ci-après : usufruit, superficie, emphytéose, zina, Houa et surélévation, habous ; »

12 Code pénal marocain :

Article 222 : «Celui qui, notoirement connu pour son appartenance à la religion musulmane, rompt ostensiblement le jeûne dans un lieu public pendant le temps du ramadan, sans motif admis par cette religion, est puni de l'emprisonnement d'un à six mois et d'une amende de 200 à 500 dirhams »

Article 418 : «Le meurtre, les blessures et les coups sont excusables s'ils sont commis par l'un des époux sur la personne de l'autre, ainsi que sur le complice, à l'instant où il les surprend en flagrant délit d'adultère »

13 "La finance islamique a été introduite au Maroc en 2007, année où la banque centrale Bank Al Maghrib a adressé aux établissements de crédit une circulaire les autorisant à commercialiser trois produits: la Mourabaha, l'Ijara et la Moucharaka, qui sont des produits financiers islamiques appelés par cette circulaire : les instruments financiers alternatifs. Des obstacles fiscaux et administratifs ont freiné le développement de ces produits" (Bellouch, 2014: 27) 
De son côté, la Cour de Cassation peut examiner les décisions rendues par les tribunaux de première instance ou par les cours d'appel en contrôlant la conformité au Droit (IEDJA, 2014).

Quant aux juridictions de proximité, celles-ci ont été créées en 201, en remplaçant les juridictions communales et d'arrondissement, mais elles ne constituent pas des juridictions sui géneris car elles dépendent des tribunaux de première instance :
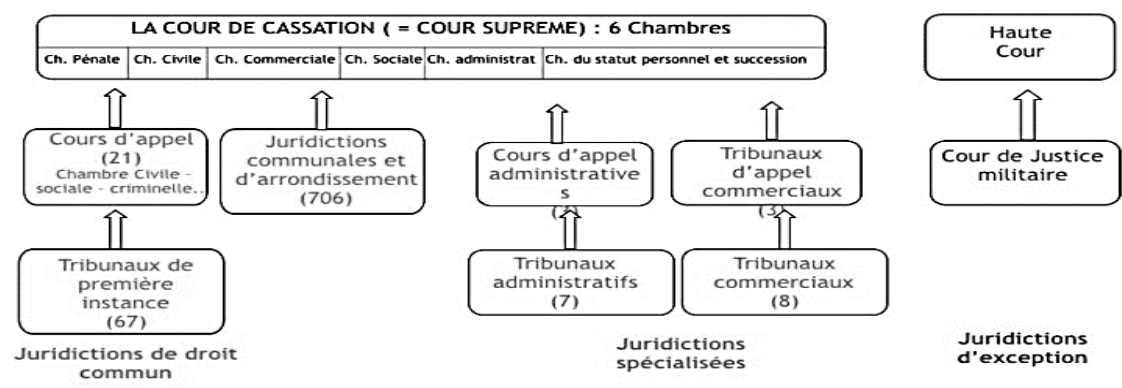

Figure 1. La Cour de Cassation. Source: IEDJA ( 2014)

Pour leur part, les juridictions spécialisées jugent les litiges qui leur sont attribués expressément par un texte spécial de loi notamment les tribunaux commerciaux et les tribunaux administratifs. Finalement, la Cour de Justice militaire représente la juridiction d'exception au Maroc.

En partant de ce contexte, il nous paraît irréfutable qu'un cours de français juridique devrait mettre le point sur les concepts juridiques provenants de deux cultures juridiques dissemblables (romaine et musulmane) véhiculées par la langue française. Par conséquent, l'enseignant ne pourrait se limiter à expliquer les implications linguistiques mais plutôt les interculturelles en prenant en considération la cohabitation de fait de deux langues/systèmes juridiques.

En bref, une bonne maîtrise de ces univers est nécessaire pour réussir le processus d'enseignement/apprentissage en offrant un meilleur épanouissement professionnel aux apprenants dans ce contexte juridique.

\subsection{Présentation et exploration du corpus}

Le corpus est un outil puissant pour enseigner les langues de spécialité. Pour cette raison, notre corpus est composé de deux textes de lois représentatifs du bijuridisme et bilinguisme du système juridique marocain: la Moudawana, diffusée en langue française, et le code civil français dans le but d' apprécier les nuances conceptuelles qui en découlent.

Tout particulièrement, nous nous attardons sur l'importance de la réforme de la Moudawana14 (code du droit de la famille marocain). Ce texte fut promulgué le 10 octobre 2004 par Le Roi Mohammed VI. Il s'agit d'un

14 La codification de ce texte fut mise en oeuvre sous le règne de Mohammed V en 1958 
nouveau code de famille qui consacre l'égalité homme-femme15 marquant une nouvelle ère juridique et à la fois un défi institutionnel et éducatif dans la société marocaine. Or, le contexte de promulgation n'était pas pacifique car l'interprétation du Coran suscitait l'affrontement entre les traditionalistes et les modernistes. Pour ces derniers, l'Islam n'accorde pas un droit d'interprétation exclusif aux Oulémas puisque la religion est une affaire entre le croyant et Allah. Ainsi, la tradition malékite, adoptée par le Royaume, est caractérisée par la modération pour soutenir le point de vue des modernistes qui plaident en faveur de la Souveraineté du roi (Commandeur des croyants) contre les aspirations d'Ijtihad des Oulémas. Inspirée par la Charia certes, mais la Moudawana est un texte juridique ouvert à l'interprétation et à la révision. (Murgue, 2011: 26)

Le choix de ces textes issus de la double culture islamique et civiliste répondrait au profil des apprenants potentiels tels que des étudiants de droit, des professionnels et/ou juristes etc. Ces apprenants doivent maitriser suffisamment le français comme langue étrangère ou bien natifs, désireux d'exercer un métier juridique au Maroc, sans oublier évidemment, les traducteurs juridiques dont la combinaison linguistique est le français.

Le déroulement de l'apprentissage contextuel sera articulé par des tâches diverses mais successives. Dans un premier temps, la tâche à accomplir serait d'exploiter la structure de ces textes pour analyser, dans un deuxième temps, les données textuelles et finalement détecter les termes ou collocations juridiques à sens ambigu ou méconnus par les apprenants.

Tout d'abord, il faut souligner que la structure interne des codes choisis participent aux mêmes notes qu'un arrêté ou loi puisqu'il s'agit d'un texte juridique. D'après Bourcier (2011), ces textes juridiques sont découpés en articles, vecteur essentiel de tout texte juridique comme l'affirme l'auteur: “ l'article est aussi au croisement du plan et du réseau des références, deux axes qui constituent l'organisation fondamentale de la connaissance juridique" Bourcier (2011, cit.par Faiz, 2006: 64).

En deuxième lieu, de ce qui est du plan linguistique, selon Faiz (2006) l'exploration de ces textes juridiques met en évidence plusieurs phénomènes importants dont elle cite:

- les difficultés de découpage correct des textes en unités d'information pertinentes,

- la complexité des structures syntaxiques des phrases dans les textes juridiques,

- l'utilisation fréquente d'anaphores (référents à des notions exprimées précédemment dans le texte via différents substituts tels que des pronoms personnels ou relatifs)

- le nombre important d'expressions propres au domaine juridique ou à d'autres domaines abordés dans les textes juridiques (par exemple, santé ou environnement),

- la variété et l'importance de termes qui sont polysémiques et «inexploitables » correctement sans une analyse contextuelle disposant d'informations complémentaires. (Faiz, 2006: 5)

15 "La Moudawana, en tant que texte conservateur inspiré des principes de la Charia apparu alors comme un texte divin, un code inaltérable. Ce code devint le symbole national de l'identité islamique du Maroc, abolissant la loi coutumière berbère pourtant légale sous le mandat français. Par cette abolition, le gouvernement marocain scella dans les textes juridiques l'unification de la nation aux principes fondateurs de l'islam" (Murgue,2011:15). 
Précisément, c'est sur ce dernier point que nous allons mettre l'accent dans le paragraphe ci-après pour explorer, si bien d'une manière non exhaustive mais représentative, les termes et/ou collocations juridiques les plus problématiques.

L'aboutissement des tâches indiquées est concrétisé par une exploitation didactique du corpus adaptée aux attentes du public en fonction de leurs besoins. Nonobstant, pour des raisons d'espace et de temps nous n'extrairons que les termes et/ou collocations les plus significatifs au niveau du contexte bijuridique marocain.

Dans le tableau ci-dessous nous avons visé quatre institutions juridiques liées au statut personnel dans chaque système juridique (marocain et français) présentant généralement une variation conceptuelle:

\begin{tabular}{|c|c|c|}
\hline $\begin{array}{l}\text { FIGURE } \\
\text { JURIDIQUE }\end{array}$ & $\begin{array}{c}\text { CONTEXTE JURIDIQUE } \\
\text { MAROCAIN (CFM)-Moudawwana }\end{array}$ & $\begin{array}{l}\text { CONTEXTE JURIDIQUE } \\
\text { FRANÇAIS (Code civil français) }\end{array}$ \\
\hline \multirow{3}{*}{$\begin{array}{c}\text { MARIAGE } \\
\text { (conditions et effets) }\end{array}$} & $\begin{array}{l}\text { Art.9 (MDWA) } \\
\text { Lorsque le Sadaq (la dot) }{ }^{16} \text { a été } \\
\text { acquitté en totalité ou en partie par le } \\
\text { fiancé, ayant servi à l'acquisition du } \\
\text { Jihaz (trousseau de mariage et } \\
\text { ameublement), (...) la perte découlant de } \\
\text { la dépréciation éventuelle du Jihaz } \\
\text { depuis son acquisition. } \\
\text { [Sadaq (صداق):la dot ;Jihaz (جاز) : } \\
\text { trousseau de mariage et ameublement] }\end{array}$ & $\begin{array}{l}\text { Art.1439 } \\
\text { La dot constituée à l'enfant commun, en } \\
\text { biens de la communauté, est à la charge de } \\
\text { celle-ci. Elle doit être supportée pour } \\
\text { moitié par chaque époux, à la dissolution } \\
\text { de la communauté, à moins que l'un d'eux, } \\
\text { en la constituant (...) }\end{array}$ \\
\hline & $\begin{array}{l}\text { Art.10 (MDWA) } \\
\text { Le mariage est conclu par consentement } \\
\text { mutuel (Ijab et Quaboul) des deux } \\
\text { contractants, exprimé en termes } \\
\text { consacrés ou à l'aide de toute expression } \\
\text { admise par la langue ou l'usage. } \\
\text { [Ijab et Quaboul (إيجاب و Gبول): } \\
\text { consentement mutuel] }\end{array}$ & $\begin{array}{l}\text { Art. } 146 \\
\text { Il n'y a pas de mariage lorsqu'il n'y a point } \\
\text { de consentement }{ }^{17} \text {. }\end{array}$ \\
\hline & $\begin{array}{l}\text { Art.84 (MDWA) } \\
\text { Les droits dus à l'épouse comportent : le } \\
\text { reliquat du Sadaq, le cas échéant, la } \\
\text { pension due pour la période de viduité } \\
(\text { Idda })^{\mathbf{1 8}} \text { et le don de consolation } \\
(\text { Mout'â })^{\mathbf{1 9}} \text { qui sera évalué en fonction } \\
\text { de la durée du mariage, de la situation } \\
\text { financière de l'époux, des motifs du }\end{array}$ & $\begin{array}{l}\text { Prestation compensatoire }^{20} \\
\text { Art.271 (Cc) } \\
\text { La prestation compensatoire est fixée } \\
\text { selon les besoins de l'époux à qui elle est } \\
\text { versée et les ressources de l'autre en tenant } \\
\text { compte de la situation au moment du } \\
\text { divorce et de l'évolution de celle-ci dans } \\
\text { un avenir prévisible. }\end{array}$ \\
\hline
\end{tabular}

16 Article 26 (Code de famille marocain): le Sadaq (la dot) consiste en tout bien donné par l'époux à son épouse, impliquant de sa part la ferme volonté de créer un foyer et de vivre dans les liens d'une affection mutuelle. Le fondement légal du Sadaq consiste en sa valeur morale et symbolique et non en sa valeur matérielle.

17 Le consentement mutuel dans le contexte juridique français est utilisé pour désigner la modalité du divorce par consentement mutuel visé aux articles 229-2 du code civil français.

18 Période pendant laquelle la femme ne peut contracter un nouveau mariage afin d'éviter confusion en matière de filiation paternelle.

19 Dédommagement versé par l'époux au profit de la femme de la femme divorcée tenant compte de la situation de celle-ci après le divorce.

20 Même si on peut l'assimiler au «don de consolation » la différence c'est que l'obligé en droit français est indistinctement les deux époux alors qu'en droit musulman l'obligé est l'époux 


\begin{tabular}{|c|c|c|}
\hline & $\begin{array}{l}\text { divorce et du degré d'abus avéré dans le } \\
\text { recours au divorce par l'époux (...). } \\
\text { [Idda (عدة): période de viduité; Mout'â } \\
\text { (متع): don de consolation] }\end{array}$ & \\
\hline \multirow{5}{*}{$\begin{array}{c}\text { DIVORCE } \\
\text { (Modalités et effets) }\end{array}$} & $\begin{array}{l}\text { Art.70 MDWA } \\
\text { Le recours à la dissolution du mariage, } \\
\text { par divorce sous contrôle judiciaire ou } \\
\text { par divorce judiciaire, ne devrait avoir } \\
\text { lieu qu'exceptionnellement et en } \\
\text { prenant en considération (...). } \\
{[\text { Tatliq (نليق) : divorce judiciaire] }}\end{array}$ & $\begin{array}{l}\text { Du divorce par consentement mutuel } \\
\text { judiciaire } \\
\text { Art. } 230 \\
\text { Le divorce peut être demandé } \\
\text { conjointement par les époux lorsqu'ils } \\
\text { s'entendent sur la rupture du mariage et ses } \\
\text { effets en soumettant à l'approbation du juge } \\
\text { une convention réglant les conséquences du } \\
\text { divorce. }\end{array}$ \\
\hline & $\begin{array}{l}\text { Art. } 78 \\
\text { Le divorce sous contrôle judiciaire est } \\
\text { la dissolution du pacte de mariage } \\
\text { requise par l'époux ou par l'épouse, } \\
\text { selon des conditions propres à chacun } \\
\text { d'eux, sous le contrôle de la justice et } \\
\text { conformément aux dispositions du } \\
\text { présent Code. } \\
{\left[\text { Talaq }{ }^{21} \text { (طلاق) : divorce sous contrôle }\right.} \\
\text { judiciaire] }\end{array}$ & $\begin{array}{l}\text { Divorce par consentement mutuel acte } \\
\text { sous signature privée contresigné par } \\
\text { avocats } \\
\text { Art.229-1 } \\
\text { Lorsque les époux s'entendent sur la } \\
\text { rupture du mariage et ses effets, ils } \\
\text { constatent, assistés chacun par un } \\
\text { avocat, leur accord dans une convention } \\
\text { (...). }\end{array}$ \\
\hline & $\begin{array}{l}\text { Art.94 (Mdwa) } \\
\text { Lorsque les deux époux ou l'un d'eux, } \\
\text { demandent au tribunal de régler un } \\
\text { différend les opposant et qui risquerait } \\
\text { d'aboutir à leur discorde (...) } \\
\text { [Chiqaq (الثقاق): Divorce judiciaire sur } \\
\text { demande de l'un des époux en raison de } \\
\text { discorde] }\end{array}$ & $\begin{array}{l}\text { Divorce accepté } \\
\text { Art. } 233 \\
\text { Le divorce peut être demandé par l'un ou } \\
\text { l'autre des époux ou par les deux lorsqu'ils } \\
\text { acceptent le principe de la rupture du } \\
\text { mariage sans considération des faits à } \\
\text { l'origine de celle-ci. Cette acceptation n'est } \\
\text { pas susceptible de rétractation, même par la } \\
\text { voie de l'appel. }\end{array}$ \\
\hline & $\begin{array}{l}\text { Art.112 (MDWA) } \\
\text { Lorsque l'époux fait serment de } \\
\text { continence à l'égard de son épouse ou } \\
\text { qu'il la délaisse, l'époux un délai de } \\
\text { quatre moi (...). } \\
\text { [Ilấ (هيلاء) : serment de continence ; } \\
\text { Hajr (هر) : délaissement] }\end{array}$ & $\begin{array}{l}\text { Divorce pour altération définitive du lien } \\
\text { conjugal } \\
\text { Art.238 } \\
\text { L'altération définitive du lien conjugal } \\
\text { résulte de la cessation de la communauté de } \\
\text { vie entre les époux, } \\
\text { lorsqu'ils vivent séparés depuis deux ans } \\
\text { lors de l'assignation en divorce }\end{array}$ \\
\hline & $\begin{array}{l}\text { Art.114 } \\
\text { Les deux époux peuvent se mettre } \\
\text { d'accord sur le principe de mettre fin à } \\
\text { leur union conjugale, soit sans } \\
\text { conditions, soit avec conditions (...). } \\
\text { [Talaq bi Itifaq (طلاق بلإتفاق) : divorce } \\
\text { par consentement mutuel] }\end{array}$ & $\begin{array}{l}\text { Divorce par faute } \\
\text { Art. } 242 \\
\text { Le divorce peut être demandé par l'un des } \\
\text { époux lorsque des faits constitutifs d'une } \\
\text { violation grave ou } \\
\text { renouvelée des devoirs et obligations du } \\
\text { mariage sont imputables à son conjoint et } \\
\text { rendent intolérable le } \\
\text { maintien de la vie commune. }\end{array}$ \\
\hline
\end{tabular}

21 Reformulation du terme « répudiation » considérée comme un privilège qui permet à l'époux de dissoudre l'union conjugale unilatéralement. Dans cette catégorie de divorce sous contrôle juidiciaire nous distinguons: le divorce révocable (Rijîi) et le divorce irrévocable (Baín) stipulés aux articles 122 et $s$. 


\begin{tabular}{|c|c|c|}
\hline & $\begin{array}{l}\text { Art.115 (MDWA) } \\
\text { Les deux époux peuvent convenir de } \\
\text { divorcer par Khol (...). } \\
\text { [Khol (الخلع): divorce moyennant une } \\
\text { compensation] }\end{array}$ & \\
\hline & $\begin{array}{l}\text { Art.163 (MDWA) } \\
\text { La garde de l'enfant }{ }^{22} \text { consiste à } \\
\text { préserver celui-ci de ce qui pourrait lui } \\
\text { être préjudiciable, à l'éduquer et à veiller } \\
\text { à ses intérêts. } \\
\text { [Hadana (الحضانة): la garde de l'enfant] }\end{array}$ & $\begin{array}{l}\text { Autorité parentale }^{\mathbf{2 3}} \\
\text { Art.61-3-1 } \\
\text { (...) Lorsque la personne est mineure, la } \\
\text { déclaration est effectuée conjointement par } \\
\text { les deux parents exerçant l'autorité } \\
\text { parentale ou par le parent exerçant seul }\end{array}$ \\
\hline & $\begin{array}{l}\text { Art.236 (MDWA) } \\
\text { Le père est de droit le tuteur légal de } \\
\text { ses enfants, tant qu'il n'a pas été déchu } \\
\text { de cette tutelle par un jugement (...) } \\
\left.\text { [Wilaya (ولاية): tutelle }{ }^{24}\right]\end{array}$ & $\begin{array}{l}\text { lautorite } \\
\text { parentale, avec son consentement } \\
\text { personnel si elle a plus de treize ans. }\end{array}$ \\
\hline \multirow{4}{*}{ FILIATION } & $\begin{array}{l}\text { Art.142 (MDWA) } \\
\text { La filiation parentale à l'égard du père } \\
\text { est légitime dans les cas où l'un des } \\
\text { motifs de la filiation paternelle existe } \\
\text { (...) } \\
{[\text { Bounouwwa (بنو): filiation paternelle] }} \\
\text { Nasab(:) : filiation parentale ; }\end{array}$ & \multirow[t]{2}{*}{$\begin{array}{l}\text { Art.314 (CC) } \\
\text { (...), la présomption de paternité se trouve } \\
\text { rétablie de plein droit si l'enfant a la } \\
\text { possession d'état à l'égard du mari et s'il n'a } \\
\text { pas une filiation paternelle déjà établie à } \\
\text { l'égard d'un tiers. }\end{array}$} \\
\hline & $\begin{array}{l}\text { Art.152 (MDWA): } \\
\text { La filiation paternelle découle: } \\
\text { 1.Rapports conjugaux (Firach) } \\
\text { 2.Aveu du père (Iqrar); } \\
\text { 3.Rapports sexuels par erreur } \\
\text { (Choubha) }\end{array}$ & \\
\hline & $\begin{array}{l}\text { Art.160 (MDWA): } \\
\text { Toute personne qui a intérêt peut } \\
\text { formuler un recours contre la véracité de } \\
\text { l'existence des conditions de la } \\
\text { reconnaissance de paternité (Istilhaq) } \\
\text { précitées } \\
\text { [Istilhaq (الإستلحاق): la reconnaissance } \\
\text { de paternité25] }\end{array}$ & $\begin{array}{l}\text { Filiation par la reconnaissance de } \\
\text { paternité ou maternité } \\
\text { Art. } 316(\mathrm{CC}) \text { : } \\
\text { Lorsque la filiation n'est pas établie dans les } \\
\text { conditions prévues à la section I du présent } \\
\text { chapitre, elle peut } \\
\text { l'être par une reconnaissance de paternité } \\
\text { ou de maternité, faite avant ou après la } \\
\text { naissance. }\end{array}$ \\
\hline & \multicolumn{2}{|l|}{ Art.153 (MDWA) } \\
\hline
\end{tabular}

22 "La garde est attribuée conjointement à la mère et au père « tant que les liens conjugaux subsistent », contrairement au droit de tutelle. En cas de divorce ou de décès du père, la garde revient à la mère (art. 171 du CFM). Elle peut néanmoins perdre son droit de garde dans certaines situations, en cas de remariage par exemple.

23 La notion d'autorité parentale en droit français est comparable à celle de la tutelle légale (du père) en droit marocain. Ainsi, la mère ne partage pas la tutelle légale avec le père" (CICADE, 2015)

24 "Lorsque le père de l'enfant est inconnu, absent, décédé ou déchu de la tutelle : la mère est à la fois détentrice du droit de tutelle et de garde, elle peut donc librement quitter le territoire marocain avec l'enfant" (CICADE :2015)

25 Ce terme désigne la reconnaissance de paternité faite par le père pourtant la reconnaissance en droit français pourrait être portée par les deux ascendants. 


\begin{tabular}{|c|c|c|}
\hline & $\begin{array}{l}\text { Les rapports conjugaux (...) suivant la } \\
\text { procédure du serment d'anathème }{ }^{26} \\
\text { (Liâane). } \\
{[\text { Liâane (اللعان) : serment d'anathème] }}\end{array}$ & \\
\hline ADOPTION & $\begin{array}{l}\text { Art.149(MDWA) } \\
\text { L'adoption } 27 \text { (Attabani) est } \\
\text { juridiquement nulle et n'entraîne aucun } \\
\text { des effets de la filiation parentale } \\
\text { légitime. } \\
\text { L'adoption dite de gratification (Jaza) } \\
\text { ou testamentaire (Tanzil), par laquelle } \\
\text { une personne est placée au rang d'un } \\
\text { héritier de premier degré, n'établit pas la } \\
\text { filiation paternelle et suit les règles du } \\
\text { testament (Wassiya). } \\
\text { [Jaza (ج) : adoption de gratification ; } \\
\text { Tanzil (تنزيل) : adoption testamentaire] }\end{array}$ & $\begin{array}{l}\text { Adoption simple et adoption plénière } \\
\text { Art.345 } \\
\text { Toutefois, si l'enfant a plus de quinze ans et } \\
\text { a été accueilli avant d'avoir atteint cet âge } \\
\text { par des personnes qui ne remplissaient pas } \\
\text { les conditions légales pour adopter ou s'il a } \\
\text { fait l'objet d'une adoption simple avant } \\
\text { d'avoir atteint cet âge, l'adoption plénière } \\
\text { pourra être demandée, si les conditions en } \\
\text { sont remplies, pendant la } \\
\text { minorité de l'enfant et dans les deux ans } \\
\text { suivant sa majorité. }\end{array}$ \\
\hline
\end{tabular}

\section{Conclusion}

Au cours de cette brève étude, nous avons réalisé que l'enseignement-apprentissage du français juridique est une mission où l'on doit prendre en considération le contexte social et interculturel dont il fait partie. Dans ce but, nous avons entamé cette étude dans une approche contextuelle et interculturelle pour montrer qu'un enseignant de FOS exerce en tant qu'intermédiaire interculturel entre le contexte juridique source et le contexte juridique cible.

Pour ce faire, des compétences sociolinguistiques (diglossie, variation dialectale) et extralinguistiques (culture, société, morale etc.) sont requises pour enseigner la culture juridique d'un pays francophone. Nous avons également vérifié qu'avant de lancer un programme/cours de français juridique, la situation plurilingue au Maroc où plusieurs langues et dialèctes coexistent, demande une attention et analyse rigoureuses autant du profil linguistique que des besoins des apprenants pour que le succès de ce processus soit garantit.

Du point de vue pratique, le choix du texte de la Moudawana est justifié aussi par son envergure historique et législative que par sa complexité terminologique. En guise d'exemple, les termes que nous avons exposés en matières de divorce (Talaq, Tatliq ...), filiation (Istilhaq, Liâane ...) et mariage (Sadaq, Îdda, Mout'â...) fortement influencées par le droit musulman.

Dans le même sens, l'exploration terminologique menée dans le chapitre 4 montre que la maîtrise de la culture juridique d'arrivée ne saurait possible qu'à travers une formation préalable des enseignants en Droit francophone comparé, dans ce cas le droit musulman et le droit français.

26 Procédure pour accuser le conjoint d'adultère comme motif de divorce en droit marocain qu'on peut verser également comme cause du divorce pour faute en droit français puisqu'il s'agit d'une violation grave des devoirs conjugaux.

27 L'adoption n'est pas reconnue au Maroc étant remplacé par l'institution de Kafala qui signifie la prise en charge affective et matérielle d'un enfant mais juridiquement ne crée pas de lien de filiation 
À ce stade, l'enseignement-apprentissage du français juridique dans un contexte bilingue et bijuridique constitue un défi continue pour les enseignants et /ou les encadrants. Ceux-ci doivent se tenir au courant de tous les amendements qui subit un concept juridique moyennant une modification de la loi dans les systèmes juridiques qui font l'objet de leur programme de formation.

En fin de compte, nous sommes conscients qu'il reste encore beaucoup d'efforts à accomplir. Les recherches dans cette ligne doivent se poursuivre dans l'objectif d'élaborer d'autres ressources ou de mettre à jour celles qui sont disponibles. Sans doute, cela aura des meilleures répercussions sur les futurs parcours pédagogiques qui miseront sur l'enseignement-apprentissage contextualisé du français juridique.

\section{Declaration of conflicting interests}

The author(s) declared no potential conflicts of interest with respect to the research, authorship, and/or publication of this article.

\section{Funding}

The author(s) received no financial support for the research, authorship, and/or publication of this article.

\section{About the author}

Khatima El Krirh holds a Phd in Translation and Interpreting studies in arabic-spanish -french languages from the University of Malaga-Spain also holds a Law Degree from UNED (spain) and actually following a course in master's Degree in law. From the defense of his doctoral thesis, she began her line of research on the complexity of legal language in different areas of knowledge: translation, linguistics and languages for specific purposes. She has also published several works on legal translation in the Arabic-Spanish-French language combination. Her teaching assignements include courses on Translation, interpreting and French lenguage. She is a member of the Literature, Image and Translation research group at the University of Cadiz.

\section{Bibliographie}

Amireault, V. (2012). La rencontre des langues et cultures en contexte d'enseignement /apprentissage du français langue étrangère dans le système universitaire chinois. Synergies: pays riverains de Mékong, 4, 51-63. < https://gerflint.fr/Base/Mekong4/amireault.pdf $>[10 / 03 / 2020]$.

Azouzi, A. (2008). Le français au Maghreb: statut ambivalent d'une langue. Synergies Europe,3, 37-50

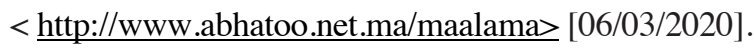

Bellouch, L.(2014). L'islam: source d'inspiration du droit marocain. Jurimast, numéro spécial,15-31

$<$ https://www.recil.grupolusofona.pt > [30/03/2020]. 
Ben Achour, I. (2007). Regard du Maghreb. Existe-t-il une culture juridique francophone? Conférence internationale des Facultés de droit ayant en commun l'usage du français (dir. Henry Roussillon). Toulouse: Presses de l'Université des sciences sociales de Toulouse,137-148.

Benzakour, F. (2012). Le français au Maroc.Une variété occultée en quête de légitimité. Ponts.Langues littératures civilisations des Pays francophones, n¹2, 114-130. <https://www.ledonline.it/index.php/Ponts/article/viewFile/455/423 > [23/02/2020].

Letafati, R et Zarei, H. (2016). Langue et traduction spécialisée. Recherche en Langue et Littérature Françaises, Revue de la Faculté des Lettres,10 (18), 85-100. <https://www.pdfs.semanticscholar.org > [5/04/2020].

Toullelon, C. (2016). L'analyse des discours de l'archéologie collectés en contexte professionnel. Points commun- Recherche en didactique des langues sur objectif(s) spécifique(s): Analyse des données et élaboration des contenus de formation en FOS : des corpus aux ressources,3 (11), 5-24.<https://www.lefrançaisdesaffaires.fr> [09/04/2020].

Damette, E. (2007). Didactique du français juridique: Français langue étrangère à visée professionnelle. Paris: Harmattan.

Dechamps C. (2013). L'enseignement/apprentissage du français juridique: une proposition de démarche terminologique et didactique. Équivalences, 40 (1-2), 191-224. <https://doi.org/10.3406/equiv.2013.1387> [25/01/2020].

El Himer, M. (2000 b). Alternance codique dans le discours des locuteurs slaouis de souche. In Dumont, P. (éd.), La coexistence des langues dans l'espace francophone, approche macrosociolinguistique, S. 1., AUPELF-UREF, pp. 253-260.

Faiz, R. (2006). Méthodologie d'aide à la structuration et à la formalisation des textes juridiques. Revue des Sciences de Gestion, Direction et Gestion 220(221), 61-71. <https://www.cairn.info/revue-des-sciences-de-gestion-2006-4-page-61.htm $\geq$ $[22 / 03 / 2020]$.

Gémar, J.C. (1998). Les enjeux de la traduction juridique: Principes et nuances. Équivalences (pp.1-19) < http://www.tradulex.com/Bern1998/Gemar.pdf > [15/03/2020].

Gémar, J.C. (1990). Les fondements du langage du droit comme langue de spécialité. Du sens et de la forme du texte juridique. Revue générale de droit., 4 (21), 717-738. < https://doi.org/10.7202/1058214ar > [30/03/2020]

Gémar, J. C. (1980). La langue juridique, langue de spécialité au Québec: éléments de méthodologie. The French Review, 6(53),880893. <https://www.jstor.org/stable/391928?read-now=1\&seq=2\#page_scan_tab_contents> [18/03/2020].

Lacámara, Ruberte, P. (2001). Les textes de spécialité : traduction professionnelle et traduction pédagogique. In Isabel Uzcanga et al. (Coord.) Presencia et renovación de la lingüística francesa (pp.187-200). <https://gredos.usal.es/bitstream/handle/10366/131465/ 978-84-7800-963301870200.pdf?sequence=1\&isAllowed=y $>$ [11/07/2020].

Mabrour, A. (2007). L'alternance codique arabe/français: emplois et fonctions. In Constellations francophones (2), 12-20. < http://www.publifarum.farum.it/ > [10/02/2020].

Magnard, R. (1990). De l'organisation judiciaire au Maroc sous le Protectorat dela France. In Petite bibliothèque,26, (1-13).< htpp://www.2a31.net>pb_numerisees >pb_26_txt> [22/03/2020].

Murgue, B. (2011). La Moudawana: les dessous d'une réforme sans précédent. In Les cahiers de l'Orient,102(15-29).

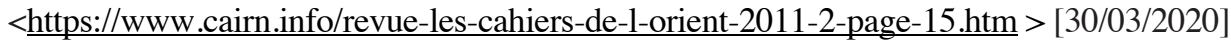

Trescases, A. (2012). La traduction juridique :un art difficile dans les pays du Maghreb. In Bilinguisme juridique dans les pays du Maghreb, colloque international (pp.116-153). Algérie: Univesité d'Alger.

Terral, F. (2004). L'empreinte culturelle des termes juridique. Meta 4 (29),876-890. <https://www.erudit.org/en/journals/meta/2004v49-n4-meta832/009787ar/> [02/03/2020] 


\section{Textes de lois et portails juridiques}

Adala.Portail juridique et judiciaire du Ministère de la Justice du Maroc (2020). Législation -Textes juridiques: Code de famille marocain <http://adala.justice.gov.ma/FR/Legislation/textesjuridiques_professionsjudiciaires.aspx> [18/04/2020]

CICADE. (2015). La tutelle et la garde en droit marocain. Droit de la famille des femmes françaises \& maghrébines. < www.cicade.org $>[31 / 03 / 2020]$.

Institut d'études sur le droit et la justice dans les sociétés arabes (2014). L'organisation juridictionnelle du Maroc < https://iedja.org/lorganisation-juridictionnelle-du-maroc/> [ 10/04/2020]

Le service public de la diffusion du droit (2020). Lois et règlements -les codes en vigueur: code civile français. < www.legifrance.gouv.fr $>$ [18/04/2020].

Secrétariat Général du Gouvernement (2020). Constitution du Maroc. < www.sgg.gov.ma.> [ 12/02/2020] 